\title{
James Webb Space Telescope Near-Infrared Spectrograph: Dark Performance of the First Flight Candidate Detector Arrays
}

\author{
Bernard J. Rauscher ${ }^{a}$, David Alexander ${ }^{a}$, Clifford K. Brambora ${ }^{a}$, Meng Chiao $^{g}$, \\ Brian L. Clemons ${ }^{a}$, Rebecca Derro ${ }^{a}$, Chuck Engler ${ }^{a}$, Ori Fox ${ }^{a, b}$, Matthew B. Garrison $^{a}$, \\ Matthew A. Greenhouse ${ }^{a}$, Greg Henegar ${ }^{a}$, Robert J. Hill ${ }^{a, c}$, Thomas Johnson ${ }^{a}$, \\ Rodolfo J. Lavaque ${ }^{a, f}$, Don J. Lindler ${ }^{a, p}$, Sridhar S. Manthripragada ${ }^{a}$, Cheryl Marshall $^{a}$, \\ Brent Mott ${ }^{a}$, Thomas M. Parr ${ }^{a}$, Wayne D. Roher ${ }^{a}$, Kamdin B. Shakoorzadeh ${ }^{a, e}$, Miles Smith $^{a}$, \\ Augustyn Waczynski ${ }^{a, f}$, Yiting Wen ${ }^{a, g}$, Donna Wilson ${ }^{a}$, Mary Ballard ${ }^{h}$ Craig Cabelli $^{h}$, \\ Edward Cheng ${ }^{h, c}$, James Garnett ${ }^{h}$, Elliott Koch ${ }^{h}$, Markus Loose $^{h}$, Majid Zandian $^{h}$, \\ Joseph Zino $^{h}$, Timothy Ellis ${ }^{i}$, Bryan Howe ${ }^{i}$, Miriam Jurado $^{i}$, Ginn Lee $^{i}$, John Nieznanski $^{i}$, \\ Peter Wallis ${ }^{i}$, James York ${ }^{i}$, Michael W. Regan ${ }^{j}$, Georgio Bagnasco $^{k}$, Torsten Böker ${ }^{k}$, Guido De \\ Marchi $^{k}$, Pierre Ferruit ${ }^{l, m, n}$, Peter Jakobsen ${ }^{k}$, and Paolo Strada ${ }^{k}$ \\ ${ }^{a}$ NASA Goddard Space Flight Center, Greenbelt, MD, U.S.A.; ${ }^{b}$ Department of Astronomy, \\ University of Virginia, P.O. Box 4000325, Charlottesville, VA, 22904, U.S.A. ${ }^{c}$ Conceptual \\ Analytics LLC, 8209 Woburn Abbey Road, Glenn Dale, MD, 20769, U.S.A.; ${ }^{d}$ Northrop \\ Grumman Technical Services, 4276 Forbes Blvd., Lanham, MD, 20706, U.S.A.; ${ }^{e}$ AK Aerospace

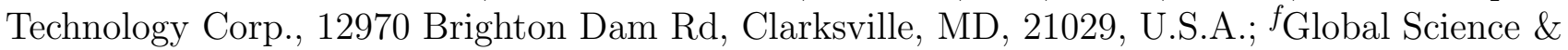 \\ Technologies, Inc., 7855 Walker Drive, Suite 200, Greenbelt, MD, 20770, U.S.A.; ${ }^{g}$ Muniz \\ Engineering Inc., 7404 Executive Place, Suite 500, Lanham, MD, 20706, U.S.A.; ${ }^{h}$ Teledyne \\ Imaging Sensors, 5212 Verdugo Way, Camarillo, CA, 93012, U.S.A.; ${ }^{i}$ ITT Space Systems \\ Division, 1447 St. Paul Street, Rochester, NY, 14653, U.S.A.; ${ }^{j}$ Space Telescope Science \\ Institute, 3700 San Martin Drive, Baltimore, MD, 21218, U.S.A.; ${ }^{k}$ ESTEC, Astrophysics \\ Division, Postbus 299, Noordwijk, NL2200 AG, Netherlands ${ }^{l}$ Université de Lyon, Lyon, \\ F-69003, France; ${ }^{m}$ Université Lyon 1, Observatoire de Lyon, 9 avenue Charles André, \\ Saint-Genis Laval, F-69230, France; ${ }^{n}$ CNRS, UMR 5574, Centre de Recherche Astrophysique \\ de Lyon; Ecole Normale Supérieure de Lyon, Lyon, F-69007, France; ${ }^{p}$ Sigma Space \\ Corporation, 4801 Forbes Blvd., Lanham, MD, 20706, U.S.A.
}

\begin{abstract}
The James Webb Space Telescope (JWST) Near Infrared Spectrograph (NIRSpec) incorporates two $5 \mu$ m cutoff $\left(\lambda_{\text {co }}=5 \mu \mathrm{m}\right) 2048 \times 2048$ pixel Teledyne HgCdTe HAWAII-2RG sensor chip assemblies. These detector arrays, and the two Teledyne SIDECAR application specific integrated circuits that control them, are operated in space at $\mathrm{T} \sim 37 \mathrm{~K}$. This article focuses on the measured performance of the first flight-candidate, and near-flight candidate, detector arrays. These are the first flight-packaged detector arrays that meet NIRSpec's challenging $6 e^{-}$rms total noise requirement.
\end{abstract}

Keywords: JWST, NIRSpec, Detectors, HgCdTe, HAWAII-2RG, H2RG

Further author information: (Send correspondence to B. J. Rauscher)

B. J. Rauscher: E-mail: Bernard.J.Rauscher@nasa.gov, Telephone: +1 301 286-4871 


\section{INTRODUCTION}

The James Webb Space Telescope (JWST) was conceived as the scientific successor to the Hubble and Spitzer space telescopes. Of all JWST "near-infrared" $(\lambda=0.6-5 \mu \mathrm{m})$ instruments, the Near-Infrared Spectrograph (NIRSpec) arguably has the most demanding detector requirements (Table 1). A team headquartered at NASA Goddard Space Flight Center (GSFC) is leading development of the NIRSpec Detector Subsystem (DS). The flight hardware is being built now by GSFC, Teledyne Imaging Systems (TIS) of Camarillo, CA, and ITT of Rochester, NY.

Table 1. Selected NIRSpec Detector Specifications ${ }^{a}$

\begin{tabular}{lcc}
\hline \hline Parameter & Required Value & Parts meet spec? \\
\hline Total noise per pixel & $<6 e^{-} \mathrm{rms}$ & Yes \\
Mean dark current per pixel & $<0.010 e^{-} s^{-1}$ pixel $^{-1}$ & Yes \\
Pixel crosstalk & $<5 \%$ & Yes \\
\hline$a$ The full set of specifications fills a substantial document. Here we highlight
\end{tabular}

those that are directly addressed in this paper.

These are exciting times for the NIRSpec DS team! The actual flight hardware is under construction, and we have some truly outstanding detector arrays vis-à-vis dark current and noise. ${ }^{*}$ In this article, we focus on the measured dark performance of recent flight candidate (FC) and near FC engineering grade (EN) sensor chip assemblies (SCA). A "flight candidate" is an SCA that has not been ruled out for flight. Readers who are interested in the overall DS architecture and detector readout patterns are referred to our previous articles and references therein. ${ }^{1-4}$

Beginning in April, 2007, TIS and the JWST Project made two incremental improvements to the near-infrared (NIR) detector design and hybridization process. Both improvements were aimed at improving the yield of flight grade parts. This directly translates to more sensitive and less expensive SCAs for JWST.

Although the details are TIS proprietary information, these improvements focused on: (1) reducing the HgCdTe lattice defect density by about an order of magnitude and (2) improving pixel interconnect operability during hybridization. Because of these improvements, parts that meet our demanding noise requirements with nearly $100 \%$ interconnect operability are being routinely produced. All three JWST NIR instruments now have flight candidate detector arrays. We believe that these improvements will directly benefit all of TIS's astronomical customers. Co-author Zandian ${ }^{\dagger}$ can provide more information for interested parties.

DS integration and test happens in different laboratories during different phases of the program. For all 3 JWST NIR instruments, ${ }^{\ddagger}$ TIS does high-volume screen testing in their Camarillo facility. They subsequently test parts that pass screen testing to acceptance level in the same facility. The JWST Project adopted this approach to maximize the degree of commonality across science instruments. An additional, although less-anticipated benefit, is that we have found it helpful from a scheduling perspective to have SCA testing integrated into the production schedules.

Upon completion of acceptance testing, flight candidates are delivered to the science instrument teams for further system level testing and detailed characterization. In the case of NIRSpec, the individual SCAs will have already been integrated into the focal plane prior to this higher level testing.

Because TIS acquires the acceptance data in their own facility, a question that we are often asked is, "who decides if a particular detector has passed?" Each instrument Principal Investigator (PI) has the final authority for his or her instrument. TIS developed the test facility and procedures in close collaboration with the instrument teams, and raw test data and detailed test reports are delivered to the teams to facilitate a decision. For NIRSpec,

\footnotetext{
*Subsequent articles will address illuminated tests including detective quantum efficiency and persistence.

${ }^{\dagger}$ mzandian@teledyne.com

${ }^{\ddagger} J W S T$ 's 3 NIR science instruments are the Near Infrared Camera (NIRCam), Fine Guidance Sensor and Tunable Filter Imager (FGS/TFI), and NIRSpec.
} 
co-authors Rauscher and Lindler routinely analyze TIS's raw data to ensure that NASA and TIS agree on the measured performance of parts. Likewise, NASA, University of Arizona NIRCam, and TIS staff have worked side-by-side in the TIS cleanroom to understand aspects of the test setups.

Following acceptance, system level testing and detailed characterization are done by the instrument teams in other facilities. For NIRSpec, NASA Goddard Space Flight Center does this higher level testing in the Detector Characterization Laboratory (DCL; see Mott et al. ${ }^{5}$ in these proceedings). This article, however, is based entirely on TIS screen and acceptance testing data. Moreover, unless otherwise noted, all analysis was done by NASA using raw data provided by TIS. We will describe DCL testing in future articles and as the data become available.

The remainder of this article is structured as follows. In Section 2, we describe the TIS test facility in which all of the data discussed here were acquired. Section 3 presents the test results.

\section{TELEDYNE TEST FACILITY}

TIS has developed an infrared detector testing facility to support production testing and flight detector selection for the JWST program. This focus puts emphasis on test throughput, repeatability, and flight documentation. The importance of test throughput is easy to see by looking at the JWST test requirements. The three instruments using $\mathrm{HgCdTe}$ detectors on JWST will be producing approximately $180 \mathrm{SCAs}$ for testing. Of these, about 20 will be selected as flight-quality. The time period for testing and flight-device selection is only about one year. Repeatability of measurements requires a rigorous program of calibration and verification, and includes cross-checking with external laboratories using both reference diode and SCA standards. To eliminate the possibility of operator variability, a highly automated system of acquisition, analysis, and reporting has been implemented. Lastly, since the SCAs are to be selected for space flight use, significant effort is spent on configuration management, environmental controls, contamination monitoring and control, and documentation.

Three cryostats perform all the testing for JWST. Each of these cryostats can accommodate up to four H2RG sensors in one cooldown. In practice, one of the SCA positions is frequently allocated to a "control" SCA or reference diode to verify test consistency. All of these cryostats are custom designs, and operated with custom electronics and software. Their internal design is such that light-tight labyrinths are included at all mechanical interfaces, consistent with the need for low-background performance at $\lambda=5 \mu \mathrm{m}\left(f<0.01 e^{-} s^{-1}\right.$ pixel $\left.^{-1}\right)$. Cooling is provided by CTI mechanical cryocoolers, with the compressors located in the mezzanine above the laboratory. Each cryostat has three separately controlled temperature zones that are cooled from a two-stage cold head. These zones provide for a $\sim 30 \mathrm{~K}$ inner radiation shield, the $77 \mathrm{~K}$ outer radiation shield, and the SCA temperature (typically $37 \mathrm{~K}$ ).

For low noise testing, the custom readout electronics are operated at a $100 \mathrm{kHz}$ per pixel readout rate and the video bandwidth is limited to about $160 \mathrm{kHz}$. The video gain of $20 \times$ and 5 Volt analog to digital converters (ADC) combine to yield a typical conversion gain of $\sim 1 e^{-} \mathrm{ADU}^{-1}$.

The cryostats have two basic configurations. The "illumination module" configuration has the SCAs viewing a short, squat diffuse-gold dome that is illuminated by internal LEDs. For each wavelength, there are 4 LEDs illuminating the dome at $90^{\circ}$ azimuthal spacing. There is enough room around the dome to place LEDs for 7 distinct wavelengths. Because the entire SCA and dome configuration can be cooled to the $37 \mathrm{~K}$ operating temperature, this configuration provides the ultimate in dark current capability. Because the LEDs are illuminating the SCAs almost directly, there is very little attenuation of the flux. Two of the three cryostats are typically used in this configuration, which is capable of demonstrating all flight requirements except for the most stringent detective quantum efficiency (DQE) measurements. These are limited by the illumination uniformity at the SCAs from this physically compact arrangement (approximately 10 to $15 \%$ variability from center to corner) and also the calibration uncertainty of the measurement (typically $\sim 5 \%$ ).

The second configuration is "GLS-4". This uses the same configuration of the cryostat as the illumination module for housing and cooling the SCAs, except that the illumination now comes from a small aperture $\sim 500 \mathrm{~mm}$ away from the SCAs. The aperture is fed by an integrating sphere, which in turn is fed by LEDs. The size of the aperture is adjusted to provide the desired intensity of illumination. There are again 7 distinct LEDs that can be commanded to illuminate the integrating sphere. Carefully designed baffles and light traps eliminate stray light. 
The GLS-4 configuration requires a second, single-stage, cold head for cooling the illumination components to $\sim 77 \mathrm{~K}$.

In normal usage, the illumination module configurations are used to screen incoming detectors for key performance parameters. The acceptance thresholds (especially for DQE) are set generously in order to avoid discarding potentially acceptable devices. The exact level depends on program requirements, taking into consideration the typical measurement accuracy of the system. After this initial screening, devices that are potentially flight-grade go through a two week period of characterization, at the end of which all performance parameters are reported. For programs requiring DQE measurements better than the $\sim 15 \%$ level, the best devices are placed in GLS-4 for DQE characterization that can take up to one week. Typical accuracies are wavelength-dependent, but are on the order of 5 to $10 \%$.

For short-wave $\left(\lambda_{\mathrm{co}}=2.5 \mu \mathrm{m}\right)$ devices, both configurations are sufficiently dark to confirm performance to JWST levels. However, because GLS-4 is large, cooling the baffles and supporting structure to less than $\sim 70 \mathrm{~K}$ is impractical. Consequently, for the mid-wave $\left(\lambda_{\mathrm{co}}=5 \mu \mathrm{m}\right)$ devices, the GLS-4 configuration is too warm to reach flight performance levels, but is more than adequate for DQE measurements.

\section{SCA TEST RESULTS}

Here we describe the measured dark properties of some of the best NIRSpec SCAs produced so far. Because SCA production is ongoing, we anticipate that the actual flight units may outperform what is reported here. Because some aspects of the GLS-4 test stations (which are used for DQE measurements) are still being validated, we focus on dark performance in this paper.

This section is structured as follows. In Section 3.1, we describe how reference pixel correction is being implemented by NASA for NIRSpec. Section 3.2 discusses crosstalk. Here we also discuss inter-pixel capacitance (IPC), and how IPC corrections to conversion gain are currently implemented by NASA for NIRSpec. Conversion gain is discussed in Section 3.3, before discussing dark current (Section 3.4) and total noise (Section 3.5). Finally, in Section 4, we close with a summary.

\subsection{Reference Pixel Correction}

In this article, we frequently refer to "reference pixel correction." Because reference pixel correction is such a common procedure, we explain how we are doing it within the NASA NIRSpec DS Team here.

For JWST, the H2RG readout integrated circuit (ROIC) uses 4 outputs. In the resulting images, pixels from each output are grouped into 4 thick $512 \times 2048$ pixel bands. The outermost 4 pixels all around the perimeter are reference pixels. Although these pixels have been designed to electrically mimic a regular pixel, they do not respond to light like a regular pixel. The reference pixels, together with the $2040 \times 2040$ regular photo-sensitive pixels, comprise the full SCA.

Early on in JWST testing at the University of Hawaii, Space Telescope Science Institute (STScI), and TIS (Rockwell Scientific at that time), it became apparent that the last 4 rows of reference pixels were typically the most stable. Here, pixels in "rows" are aligned in the fast scan direction. This insight informed the reference pixel procedure that was subsequently adopted by the University of Hawaii, STScI, and now the GSFC NIRSpec DS Team. Although TIS continues to experiment with other methods, for this article, the following procedure was used.

For each of the four outputs, iterative sigma clipping is used to compute a mean reference pixel value. We use the IDL DJS_ITERSTAT procedure with a $3 \sigma$ clipping threshold. This value is subtracted from every pixel in the corresponding output. Although this procedure does not remove higher frequency $1 / f$ noise that appears as horizontal banding in frames, it has proven to be very robust against the most common detector artifacts. Using this procedure with good SCAs, we meet NIRSpec's total noise requirements with margin (see Section 3.5). 


\subsection{Crosstalk}

We define crosstalk, $\zeta$, as the ratio of the charge that appears in a neighboring pixel to that appearing in the pixel of interest. Crosstalk includes electrical (e.g. cable settling, inter-pixel capacitance) and electro-optical components (e.g. charge diffusion in the $\mathrm{HgCdTe}$ ). Our normalization is such that the pixel that was struck is identically equal to one. In the following, we report crosstalk in $3 \times 3$ pixel grids of pixels like the following,

$$
\text { Example } \zeta=\left(\begin{array}{ccc}
0 & \zeta_{y} & 0 \\
\zeta_{x} & 1 & \zeta_{x} \\
0 & \zeta_{y} & 0
\end{array}\right)
$$

In Equation 1, we explicitly allow for different crosstalk in the $x$ and $y$ directions because this behavior is often observed in H2RG SCAs. Although additional asymmetries are also observed, they are usually small compared to the primary vertical/horizontal asymmetry. One important form of crosstalk that is not explicitly accounted for in this simple $x y$ symmetry is electrical crosstalk due to time constants in cable harnesses. This appears as charge trailing in the readout direction.

For NIRSpec's $\lambda_{\mathrm{CO}}=5 \mu \mathrm{m}$ detector arrays, and for photons striking the centers of pixels, crosstalk is completely dominated by IPC. When off-center photons are considered, other effects including charge diffusion, can become important. For simplicity, we neglect these illumination dependent effects here.

One important application of crosstalk measurements is making IPC corrections. Fox et al. ${ }^{6}$ discuss IPC in $\mathrm{HgCdTe}$ detector arrays in detail. We use the same methodoloy here. Crosstalk is closely related to the $\alpha$ and $\beta$ terms in the detector point spread function (PSF) that is used to make IPC corrections to conversion gain. For the symmetry shown in Equation 1, the IPC PSF appears as follows,

$$
\mathrm{IPC} \operatorname{PSF}=\left(\begin{array}{ccc}
0 & \alpha & 0 \\
\beta & 1-2(\alpha+\beta) & \beta \\
0 & \alpha & 0
\end{array}\right) .
$$

From Equations 1 and 2, we see that crosstalk can be related to the IPC terms as follows,

$$
\begin{aligned}
\alpha & =\frac{\zeta_{y}}{1+2\left(\zeta_{x}+\zeta_{y}\right)}, \text { and } \\
\beta & =\frac{\zeta_{x}}{1+2\left(\zeta_{x}+\zeta_{y}\right)} .
\end{aligned}
$$

For NIRSpec, crosstalk to nearest neighbors has been computed using the following three techniques: (1) hot pixel neighbors, (2) naturally occurring cosmic rays, and (3) autocorrelation. TIS also measures crosstalk for JWST using the H2RG's individual pixel reset capability to program grids of pixels to different values than their neighbors. This technique agrees well with crosstalk measured using methods 1 and 3 . Crosstalk measured using cosmic rays tends to be somewhat higher. In the following paragraphs, we briefly describe techniques 1-3 with reference to flight candidate SCA H2RG-S047. Most other good quality NIRSpec SCAs have had crosstalk properties nearly identical to this part. The interested reader should contact co-author Cabelli ${ }^{\S}$ for more information on the TIS pixel grid technique.

\subsubsection{Hot Pixel Neighbors}

The hot pixel neighbors technique searches multiple short correlated double sampling (CDS) integrations for isolated, unsaturated, positive statistical outliers. These "hot pixels" are directly used to compute $\zeta$ to each of their neighbors. The raw data consist of a set of 50 MULTI- $88 \times 1$ sampled dark integrations (see Rauscher et $a .^{3}$ for a discussion of NIRSpec readout modes).

We begin by applying a reference pixel correction (see Section 3.1) to every frame of every ramp. We then form the median of all 50 ramps, and determine slopes by 2-parameter least squares line fitting. ${ }^{3}$ Hot pixels are

\footnotetext{
${ }^{\S}$ ccabelli@teledyne.com
} 
selected between two thresholds. These are typically $0.5 e^{-} s^{-1}$ up to a few electrons per second. The upper threshold is chosen to exclude saturated pixels. From this collection of hot pixel candidates, we select those that are isolated in $7 \times 7$ pixel neighborhoods, and renormalize them so that the central pixel has the value of 1 . Finally, we form the pixel-by-pixel median of each pixel in the sub-image. The results for SCA H2RG-S047 are typical (Equation 5). The slightly higher crosstalk immediately to the right of the hot pixel is almost certainly due to electrical crosstalk in the readout electronics.

$$
\text { Hot pixel } \zeta=\left(\begin{array}{ccc}
0.001 & 0.011 & 0.001 \\
0.012 & 1 & 0.018 \\
0.00075 & 0.011 & 0.001
\end{array}\right)
$$

\subsubsection{Cosmic Rays}

In the cosmic ray technique, symmetric, isolated, cosmic ray events are used to measure crosstalk. The input data are the same data set as for the hot pixel neighbors technique described above.

Following reference pixel correction (see Section 3.1), we subtract dark current by subtracting the median of all 50 dark ramps from every individual ramp. This yields a set of 50 dark subtracted ramps. Because the reset anomaly $^{3}$ can cause false detections, we discard the first 4 frames of every ramp. Each ramp is processed as a set of EXPTIME $=10.62 s$ dark CDS integrations. In other words, and using IDL array indexing conventions, we form CDS images as follows; $\operatorname{CDS} 5=\mathrm{d} \operatorname{In}[*, *, 5]-\mathrm{d} \operatorname{In}[*, *, 4], \operatorname{CDS} 6=\mathrm{d} \operatorname{In}[*, *, 6]-\mathrm{d} \operatorname{In}[*, *, 5]$, etc. Within any one ramp, these CDS images are used to scan for cosmic ray events.

In searching for cosmic rays, it is important to exclude potential false positives including hot pixels, double cosmic ray hits to the same pixel in a ramp, and random telegraph noise ${ }^{1,3,7}$ (RTN). We begin by masking out hot pixels, and then search the remaining pixels for CDS images in which the integrated charge exceeds $400 e^{-}$. We furthermore ensure that in any one ramp, candidate pixels experience only one significant positive transition (to rule out multiple cosmic ray hits on one pixel), and no significant negative transitions (to rule out RTN). We furthermore exclude any pixels for which there is a hot pixel in a $7 \times 7$ pixel region, and any saturating events.

For the remaining pixels in the sample, we do a 3-parameter least squares fit, allowing for one slope and two zero points. The difference between the zero points is the amplitude of the cosmic ray hit. We do this for the pixel that was struck, as well as for it's neighbors. We renormalize the cosmic ray amplitude maps, and then compute the modal value of each pixel. Equation 6 shows the resulting crosstalk grid for SCA H2RG-S047. This result is typical for NIRSpec SCAs.

$$
\text { Cosmic ray } \zeta=\left(\begin{array}{ccc}
0.004 & 0.022 & 0.004 \\
0.025 & 1 & 0.027 \\
0.004 & 0.021 & 0.0043
\end{array}\right)
$$

Crosstalk computed using cosmic rays is usually slightly higher than that computed using other techniques. This happens because cosmic rays couple to their neighbors by lateral charge diffusion within the $\mathrm{HgCdTe}$, as well as by IPC. Moreover, because events are not necessarily centered in pixels, there is some blurring.

\subsubsection{Autocorrelation}

The autocorrelation technique follows Moore et al. ${ }^{8,9}$ in using noise correlations in pairs of CDS integrations to measure the IPC coupling to neighbors. Unlike the previous two techniques, the autocorrelation method requires illuminated integrations. At TIS, the noise-squared signal XGAIN ramps are ideal. In an XGAIN ramp, the SCA is illuminated so that pixels are filled to about $10^{4} e^{-}$at the end of each MULTI- $10 \times 1$ integration. There are typically 50 integrations in an XGAIN sequence.

We begin by using two ramps of the MULTI- $10 \times 1$ sampled XGAIN data (10 frames per ramp), construct pairs of CDS images, and then form the difference, D. The following is an example.

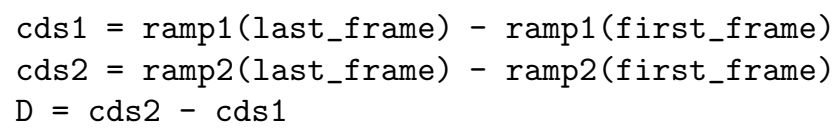


We create a good pixel mask by: (1) deleting hot pixels, (2) deleting pixels near the edges of each output, and (3) deleting $3 \sigma$ statistical outliers. For purposes of implementing the autocorrelation method, the hot pixel threshold is typically $>0.4 e^{-} s^{-1}$ pixel $^{-1}$. We use the IDL DJS_ITERSTAT procedure with sigrej $=3$ to delete statistical outliers.

There is some electromagnetic interference (EMI) in the TIS test setups. Although the EMI is small and included in all noise measurements reported in this paper, it can adversely affect the noise correlation that is important here. For this reason, we often apply a gentle high-pass filter in the time domain to remove frequencies lower than about $200 \mathrm{~Hz}$. If this step is deleted, the results are not substantially altered.

We select pixels that are good, and for which all neighbors in a $7 \times 7$ region are also good. For each of these, we compute the autocorrelation matrix. This procedure is repeated for the 25 sequential pairs of ramps in the XGAIN experiment. The final autocorrelation matrix is the median of the 25 results. The IPC $\alpha$ term is computed from the median autocorrelation matrix, $R$, and the formulae given in Moore's ${ }^{9}$ Figure 2. For example, and using IDL array index conventions, we proceed as follows,

$$
\begin{aligned}
c & =\frac{R[1,0]+R[0,1]}{2 R[0,0]}, \text { and } \\
\alpha & =\frac{1}{2} c-c^{2} .
\end{aligned}
$$

In Equation 7, pixel $[0,0]$ is the center value of the measured autocorrelation matrix. Equation 8 is valid to second order in $\alpha$ and $c$.

Following Moore et al., ${ }^{8,9}$ and for fully-symmetric IPC crosstalk, the IPC corrected variance of brightly illuminated integrations is related to the measured variance as follows,

$$
\sigma_{\text {true }}^{2}=(1+8 \alpha) \sigma_{\text {meas. }}^{2} .
$$

Equation 10 shows a typical crosstalk matrix that was computed using the autocorrelation technique. In this case, the SCA is FC grade device H2RG-S047.

$$
\text { Autocorrelation } \zeta=\left(\begin{array}{ccc}
0 & 0.015 & 0 \\
0.015 & 1 . & 0.015 \\
0 & 0.015 & 0
\end{array}\right)
$$

\subsection{Conversion Gain}

Conversion gain, $g_{c}$, relates the number of electrons on the integrating node to the number of analog to digital converter units (ADU) recorded by the detector system. Here we report $g_{c}$ in units of $e^{-} / \mathrm{ADU}$. For relatively high fluence exposures, we use the "noise-squared signal" or "photon transfer" ${ }^{10}$ technique.

In TIS HAWAII series detector arrays, the noise-squared signal technique often over-estimates $g_{c}$ for signals near the dark current floor. The cause is usually attributed to changes in pixel capacitance as charge integrates in the depletion region of individual $\mathrm{HgCdTe}$ diodes. Many groups (e.g. U. Hawaii, STScI, TIS) typically measure transimpedance gains of about $4 \mu \mathrm{V} / e^{-}$in $\lambda_{\mathrm{co}} \sim 5 \mu \mathrm{m}$ detector arrays under $300 \mathrm{mV}$ of reverse bias and near the dark current floor. For signal levels near mid-well, this can degrade to about $3.4 \mu \mathrm{V} / e^{-}$(based on TIS analysis). Because $g_{c}$ scales inversely with transimpedance gain, we expect $g_{c}$ computed for dark conditions to be lower than for signal levels near mid well. Our own spot checks suggest that the transimpedance gain of SCA H2RG-S042 degrades by about $12 \%$ for signal levels near $1.5 \times 10^{4} e^{-}$.

It is therefore important to compute conversion gain for signals close to those that are being calibrated. In practice, the noise-squared signal technique works well for brightly illuminated tests such as QE. On the other hand, for dark tests including read noise, total noise, and dark current, $g_{c}$ needs to be computed for signal levels near the dark current floor of the detector array under test. All of the dark current, total noise, and read noise results that are reported in this article therefore use conversion gains computed using the "noise modeling" technique that is described in Section 3.3.2. 
Although space limitations prevent us from making detailed comparisons between noise-squared signal and noise modeling techniques here, we believe that the measured transimpedance gains (Table 2) are completely consistent with those measured by other groups for comparable devices biased for the same $\sim 10^{5} e^{-}$well depth. For our purposes, one compelling advantage of the noise modeling technique is that the same data set can be used to compute $g_{c}$, dark current, and total noise. Another is that no extrapolation is needed, as would be the case using noise-squared signal, to compute $g_{c}$ for dark current.

Finally, and although we do not discuss the topic here, we believe that ${ }^{55} \mathrm{Fe}$ x-rays will provide a convenient and robust way to measure conversion gains at moderately low signal levels within about a year. Fox et al. ${ }^{6}$ describe recent measurements of the pair creation energy in $\lambda_{\text {co }} \sim 1.7 \mu \mathrm{m}$ HST WFC3 SCAs. They are in the process of extending the ${ }^{55} \mathrm{Fe}$ x-ray technique to $\lambda_{\mathrm{co}} \sim 2.5$ and $5 \mu \mathrm{m}$ detector arrays now.

\subsubsection{Noise-Squared Signal}

The noise-squared signal technique uses shot noise on integrated charge to derive conversion gain. For CDS integrations, and considering only read noise and shot noise on integrated charge, total noise is related to read noise per CDS as follows,

$$
\sigma_{\text {total }}^{2}=\sigma_{\mathrm{CDS}}^{2}+\frac{1}{g_{c}} S
$$

In this expression, $S$ is the integrated signal per pixel. Conversion gain can therefore be measured by plotting total noise squared as a function of signal and fitting a straight line. The conversion gain, $g_{c}$, is the reciprocal of the fitted slope.

In H2RG SCAs, IPC tends to filter out some of the noise, and the noise-squared signal technique tends to over-estimate the conversion gain. For this reason, it is customary to apply an IPC correction to recover the "true" variance. The interested reader is referred to Moore's ${ }^{8,9}$ articles that first described the effect for more information. In JWST H2RGs, IPC tends to reduce $g_{c}$ by about $12 \%$ compared to what is measured using the noise-squared signal technique. A similar IPC correction is also applied in the noise modeling technique that is described in the next section.

\subsubsection{Noise Modeling}

As is the case in the noise-squared signal techniqe, noise modeling attempts to model the noise in integrations including read noise and shot noise on integrated flux. Noise modeling differs from noise-squared signal in that multiple non-destructive reads are used to make measurements near the noise floor of the device under test. In Rauscher et al., ${ }^{3}$ we presented a model for how noise averages down using multiple non-destructive reads,

$$
\sigma_{\text {total }}^{2}=\frac{12(n-1)}{m n(n+1)} \sigma_{\text {read }}^{2}+\frac{6\left(n^{2}+1\right)}{5 n(n+1)}(n-1) t_{g} f-\frac{2(2 m-1)(n-1)}{m n(n+1)}(m-1) t_{f} f .
$$

Here, $\sigma_{\text {total }}$ is the total noise in units of $e^{-}$rms, $\sigma_{\text {read }}$ is the read noise per frame in units of $e^{-}$rms, and $f$ is flux in units of $e^{-} s^{-1}$ pixel $^{-1}$, where $f$ includes photonic current and dark current. The noise model includes read noise and shot noise on integrated flux, which is correlated across the multiple non-destructive reads samplingup-the-ramp. For the special case of dark integrations, $f=i_{\text {dark }}$. This expression was validated using real and simulated data. Interested readers are referred to our earlier paper ${ }^{3}$ for the details.

If the only source of signal is dark current, and read noise and dark current are expressed in instrumental ADU units, Equation 12 can be rewritten as follows,

$$
\sigma_{\text {total }}^{2}=\frac{12(n-1)}{m n(n+1)} \sigma_{\text {read }}^{2}+\frac{6\left(n^{2}+1\right)}{5 n(n+1)}(n-1) g_{c}^{-1} t_{g} i_{\text {dark }}-\frac{2(2 m-1)(n-1)}{m n(n+1)}(m-1) g_{c}^{-1} t_{f} i_{\text {dark }} .
$$

When large data sets are available and the dark current is known in instrumental ADU/s units, Equation 13 can be used to measure read noise and conversion gain. Each TIS dark data set contains 50 MULTI- $88 \times 1$ sampled EXPTIME $=923.94 s$ dark integrations. In practice, we find this to be more than adequate for measuring $g_{c}$ so long as the reset anomaly and temporal variations in the noise properties of the test setup are correctly handled. 

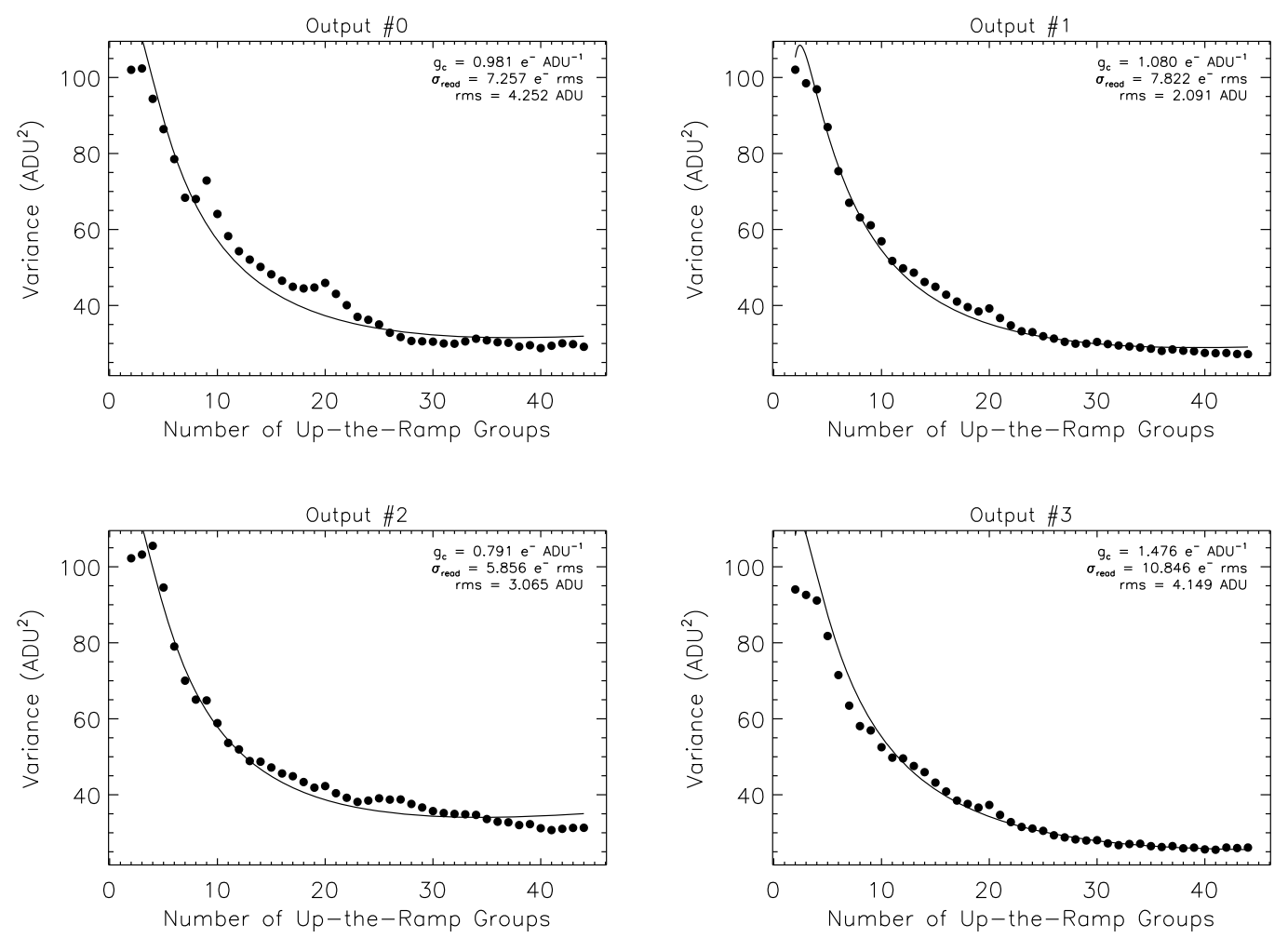

Figure 1. Modeling the noise in a pair of sampled-up-the-ramp darks as a function of the number of non-destructive samples, $n$. The solid line is the least-squares fit of Equation 13 to the data. The fitting parameters are $\sigma_{\text {read }}$ and $g_{c}$. For any one ramp pair, the uncertainties in fitted parameters are large because of time dependent noise in the test setup. When many ramp pairs are modeled, the distributions of fitted parameters are well-behaved, and the derived $g_{c}$ values are consistent with those measured using more traditional methods.

The reset anomaly is characterized by non-linearity in the early frames following pixel reset. Although the reset anomaly appears to be unrelated to response linearity, these early frames nonetheless fall below below a line projected through the later, asymptotic portion of the ramp. In Rauscher et al., ${ }^{3}$ we presented a technique for calibrating out the reset anomaly. Here, because of an abundance of data, we simply discard the first half of each ramp, and work only with the later asymptotic samples. In other words, we throw away the first half of each ramp and treat the TIS data as a collection of 50 MULTI- $44 \times 1$ sampled EXPTIME $=456.66 s$ dark integrations. In the resulting data set, there are 1225 distinct pairs of integrations. Each pair of integrations can be used to model how noise averages down as $n$ varies within the range $2 \leq n \leq 44$. Least-squares fitting was done using the IDL AMOEBA procedure. The free parameters were $g_{c}$ and $\sigma_{\text {read }}$.

Figure 1 shows the resulting noise model for a single pair of typical ramps. The fit is not perfect, and in this case the fit over-shoots the data points for small $n$. For other pairs of ramps, the reverse is true, and for any one pair of ramps the uncertainty in the fitted values of $\sigma_{\text {read }}$ and $g_{c}$ is large. However, when many ramp pairs are modeled, the resulting distributions of fitted parameters are well behaved (see Figures 2 and 6).

We have used the noise modeling technique to compute $g_{c}$ and transimpedance gain for all SCAs discussed in this paper (Table 2). These figures incorporate an IPC correction that was computed using Equation 9, and with $\alpha=0.015$ for all SCAs. These gains are entirely consistent with the known H2RG design values for transimpedance gain. Moreover, the measured conversion gains are nearly identical to what other labs have measured for $J W S T \lambda_{\text {co }} \sim 5 \mu \mathrm{m}$ SCAs under similar bias and ultra-low background conditions.

Formally, the noise modeling technique has very small random errors. A typical $g_{c}$ histogram has a standard deviation of about $0.3 e^{-} \mathrm{ADU}^{-1}$. Because the histogram is made from 1225 dark ramp pairs, the uncertainty in the mean is about $0.01 e^{-} \mathrm{ADU}^{-1}$. These uncertainties are probably comparable to those associated with the IPC 


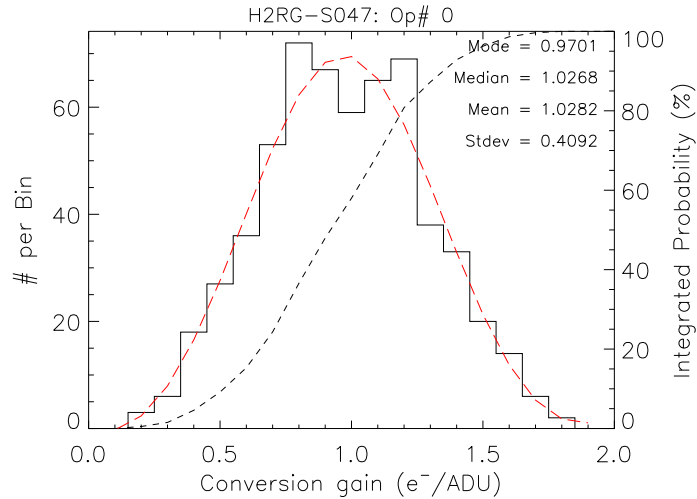

a)

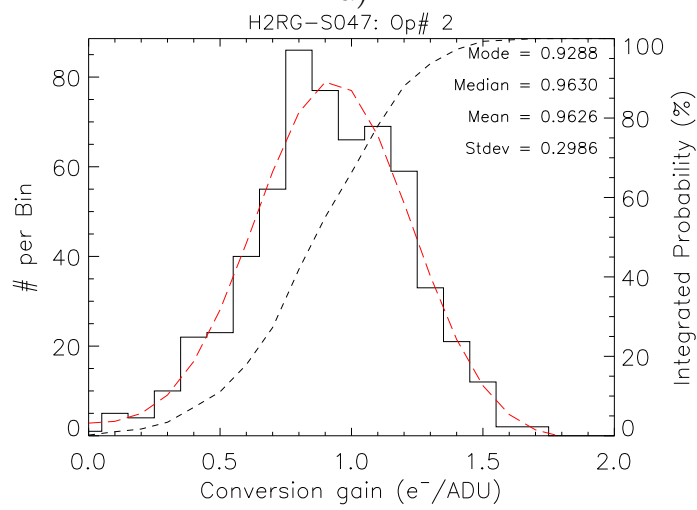

c)

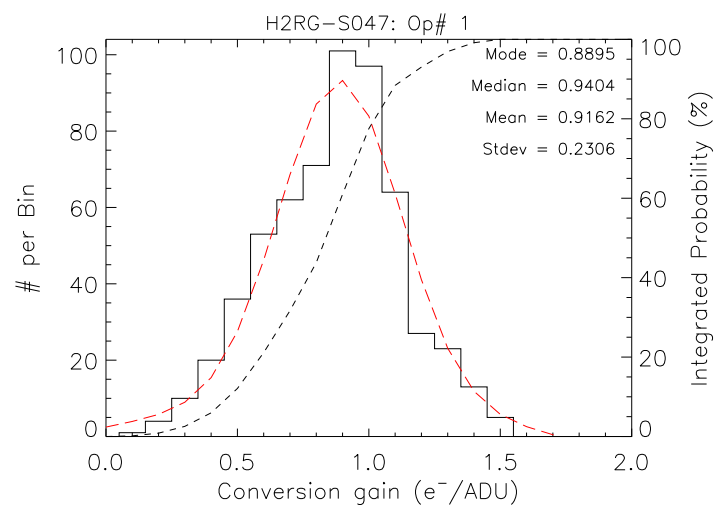

b)

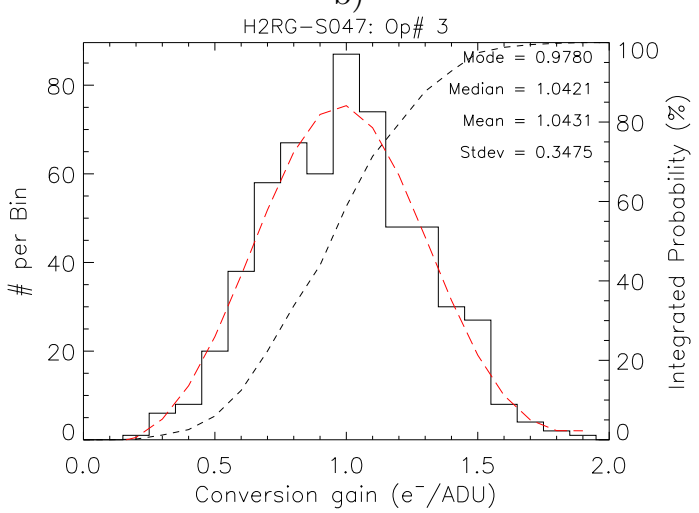

d)

Figure 2. FC grade SCA H2RG-S047: Histograms of $g_{c}$ computed by modeling noise in pairs of sampled-up-the-ramp darks.

Table 2. Conversion and Transimpedence Gains: Dark

\begin{tabular}{|c|c|c|c|c|c|c|c|c|c|c|}
\hline \multirow[b]{2}{*}{ SCA } & \multicolumn{5}{|c|}{$\begin{array}{l}\text { Conversion Gain by Output }{ }^{a, b} \\
\left(e^{-} \mathrm{ADU}^{-1}\right)\end{array}$} & \multicolumn{5}{|c|}{$\begin{array}{l}\text { Transimpedence Gain by Output } \\
\qquad\left(\mu \mathrm{V} / e^{-}\right)\end{array}$} \\
\hline & $\# 0$ & $\# 1$ & $\# 2$ & $\# 3$ & Mean & $\# 0$ & $\# 1$ & $\# 2$ & $\# 3$ & Mean \\
\hline H2RG-S042-IR50-ITT & 0.86 & 1.00 & 1.01 & 1.16 & 1.01 & 4.44 & 3.81 & 3.78 & 3.29 & 3.83 \\
\hline H2RG-S044-IR50-ITT & 1.03 & 0.80 & 1.07 & 0.98 & 0.97 & 3.70 & 4.77 & 3.56 & 3.89 & 3.98 \\
\hline H2RG-S047-IR50-ITT & 1.03 & 0.94 & 0.96 & 1.04 & 0.99 & 3.70 & 4.06 & 3.97 & 3.67 & 3.85 \\
\hline
\end{tabular}

${ }^{a}$ Video gain $=40 \times$, video bandwidth $=160 \mathrm{kHz}$, bias for $6 \times 10^{4} e^{-}$well depth

${ }^{b}$ The reported value is the median of pixel values.

${ }^{c}$ Referred to the SCA output amplifiers. 
correction, which we take to be the difference between the hot pixel and autocorrelation techniques, $\sigma_{\alpha} \approx 0.003$. When uncertainties in the IPC determination are included, we believe that the conversion gains computed using noise modeling are accurate to within about $\sigma_{g_{c}} \approx \pm 0.02 e^{-} \mathrm{ADU}^{-1}$. However, these low uncertainties are realized only for signals very close to the dark current floor. Further study is needed to understand how these uncertainties in the mean value of $g_{c}$ apply to individual pixels.

In the months ahead, we intend to further validate these methods by extrapoling measurements from the noise-squared signal technique to very low light levels at TIS. When the flight hardware arrives at GSFC, more sophisticated validation will be possible using DCL test setups that facilitate noise-squared signal measurements under ultra-low background conditions.

\subsection{Dark Current}

Dark current is the signal that accumulates in pixels under dark conditions. At NIRSpec's T $\sim 37 \mathrm{~K}$ operating temperature, the dominant components are quantum mechanical tunneling from traps and parasitics associated with ROIC operation in the overall context of the DS. The DS "context" includes cable harnesses and fanout circuitry on the ROIC package that are driven by ROIC amplifiers. Because of the interplay between readout mode, the DS system, and dark current, it is important to measure dark current using a readout mode and system that are representative of the end application. In the case of NIRSpec, we therefore measure dark current using the baseline MULTI- $22 \times 4$ readout pattern, even though this almost certainly generates more dark signal than would be seen using fewer up-the-ramp samples. During NIRSpec integration and test (I\&T), we will study how total noise trades with readout mode. At that time, we may change the baseline. For now, however, TIS is measuring dark current using MULTI- $22 \times 4$ integrations.

The actual dark current experiment contains 50 MULTI- $22 \times 4$ integrations that are taken only after the SCA has been cold and dark for about 3 days. Likewise, we avoid exposing the SCA to bright light for about 24 hours before beginning the experiment to rule out long timescale persistence. Even with these precautions, we do occasionally see persistent relics in the first few ramps. For this reason, the actual dark current of the SCAs is probably somewhat less than is reported here.

The data are calibrated as follows. For each ramp of the 50, we compute pixel slopes using 2-parameter least squares fitting. The slope images are multiplied by the integration time to form 50 integrated signal images. A reference pixel correction is applied to each of the 50 integrated signal images. Finally, the images are stacked using iterative sigma clipping for each pixel. Iterative sigma clipping is implemented using the IDL DJS_ITERSTAT procedure with a $3 \sigma$ threshold. The resulting dark map, in units of ADU, is converted to electrons per second per pixel using the $g_{c}$ values given in Table 2. Figure 3 shows the dark current maps for all SCAs discussed in this paper. Figures 4 and 7 show the dark current histograms for each of these parts.

\subsection{Total Noise}

Total noise is the noise, including all noise components that an astronomer would see, in a baseline MULTI- $22 \times 4$ integration. Total noise includes any noise that may be generated by the SCA, cables, and readout system. It includes any EMI that may be present in the test setup. The only noise components that are excluded are due to transients (e.g. cosmic rays). For an operable pixel, total noise and DQE are probably the two parameters that most directly affect NIRSpec sensitivity.

At TIS, total noise is measured using the same set of 50 dark ramps that was used to measure dark current. Calibration is also broadly similar to what is done in the dark current experiment. 2-parameter least-squares fitting is used to find the slope of each pixel. These slope images are multiplied by the integration time to make 50 integrated signal images. After applying a reference pixel correction to each signal image, the images are stacked using iterative sigma clipping. As was the case for dark current, we typically use the IDL DJS_ITERSTAT procedure with a $3 \sigma$ clipping threshold. In addition to computing the mean of each pixel, DJS_ITERSTAT can compute the standard deviation of each pixel. The total noise map is made using these standard deviations. Finally, the total noise map is converted from ADU to electrons using the conversion gains listed in Table 2. Figures 5 and 8 show the total noise histograms for all parts discussed in this article. 
H2RG-S042

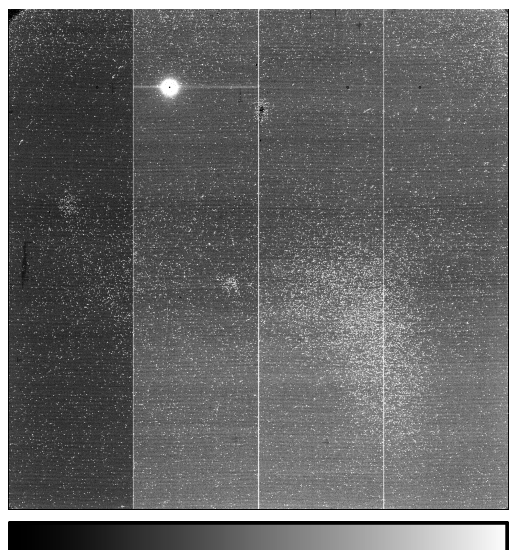

$0.000 \quad e^{-/ s} /$ pixel $\quad 0.020$

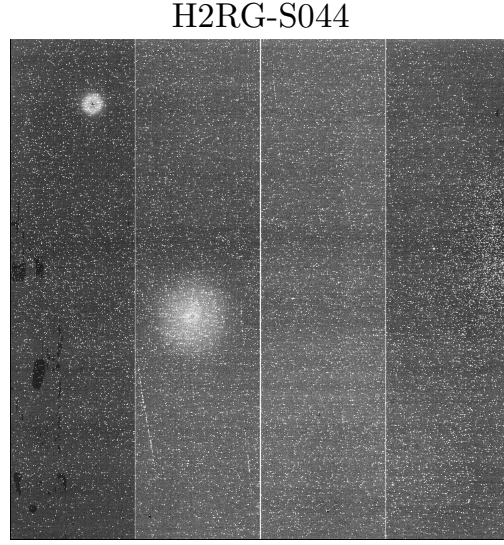

$0.000 \quad e^{-} / s /$ pixel $\quad 0.020$

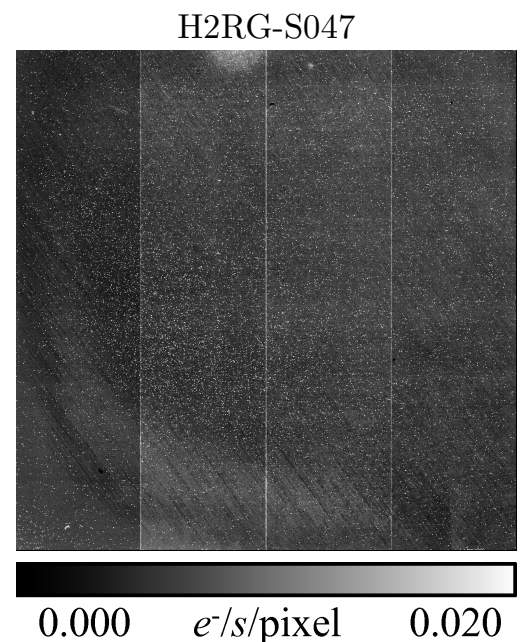

Figure 3. Dark current maps for all parts discussed in this article. The grayscale is from 0 to $0.020 e^{-} s^{-1}$ pixel $^{-1}$. H2RG-S042 has a photo-emissive defect (PED). For this reason, it is not FC grade. The two circular bright areas in H2RG-S044, and the semi-circular bright area along the top of H2RG-S047 are persistence from an earlier experiment. Although we see persistence in these darks, the available data for these and other $J W S T$ parts suggests that our $0.1 \%$ persistence specification will be met. Persistence, along with DQE and other illuminated tests, will be discussed in future articles.

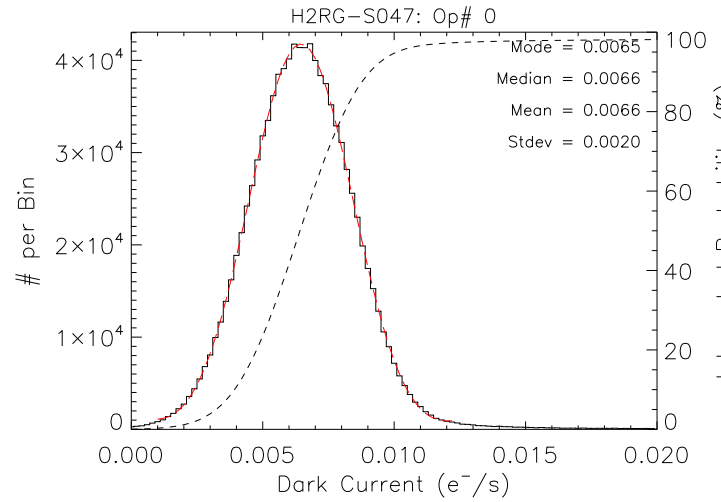

a)

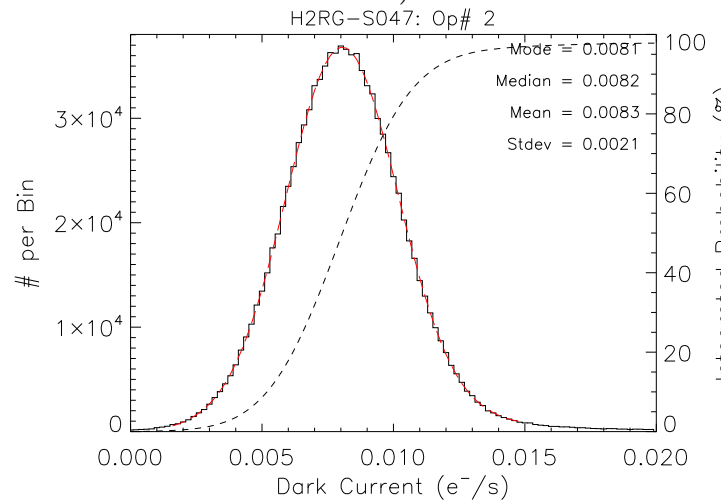

c)

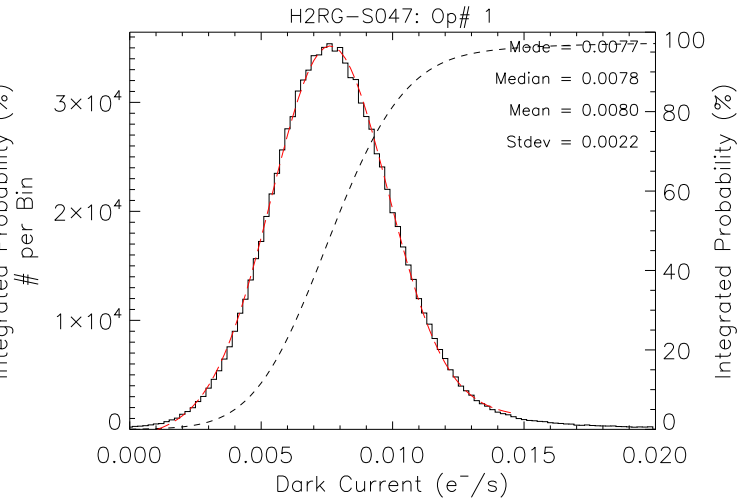

b)

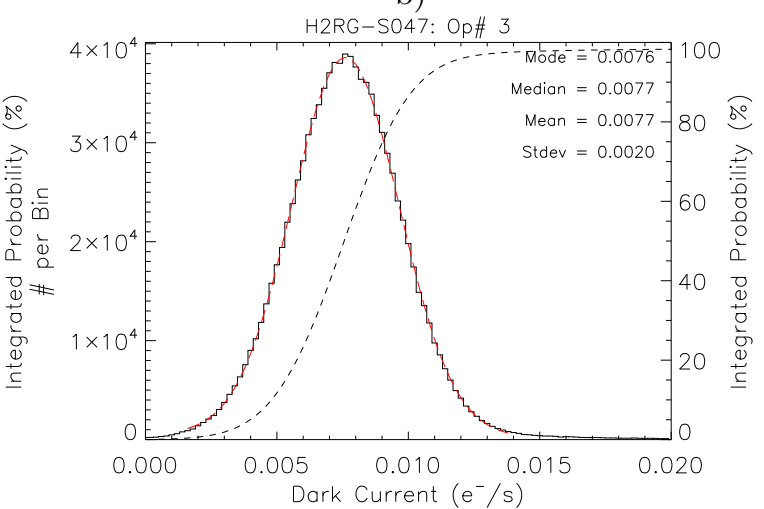

d)

Figure 4. Dark current per output of flight candidate SCA H2RG-S044. These per-pixel histograms were made using a set of 50 EXPTIME $=892 s$ MULTI- $22 \times 4$ sampled integrations. Note that dark current, as defined here, includes the reset anomaly ${ }^{3}$ and any ROIC glow that may be present. 


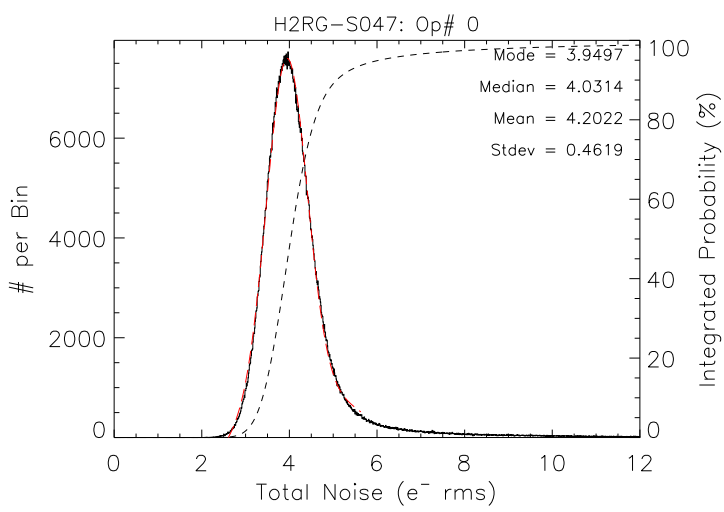

a)

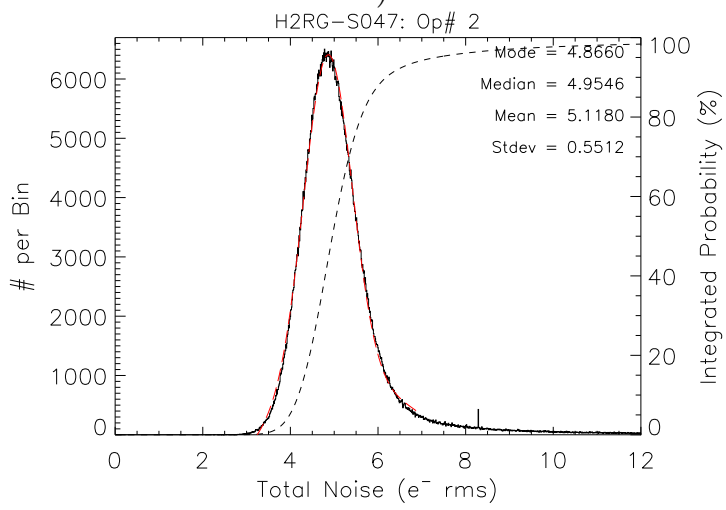

c)

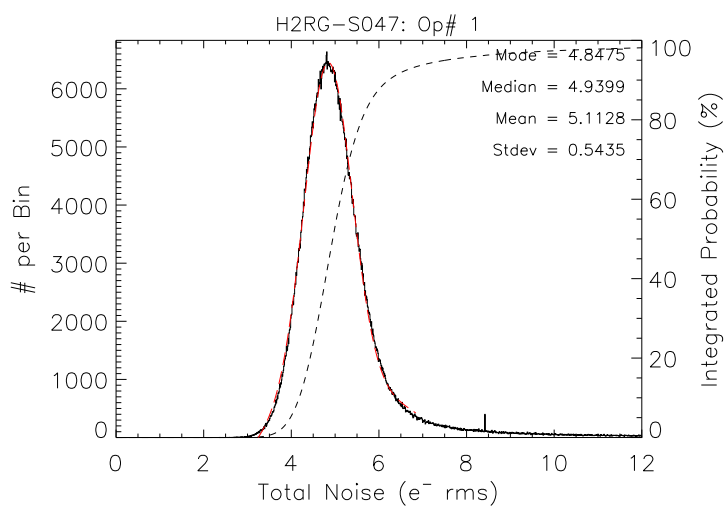

b)

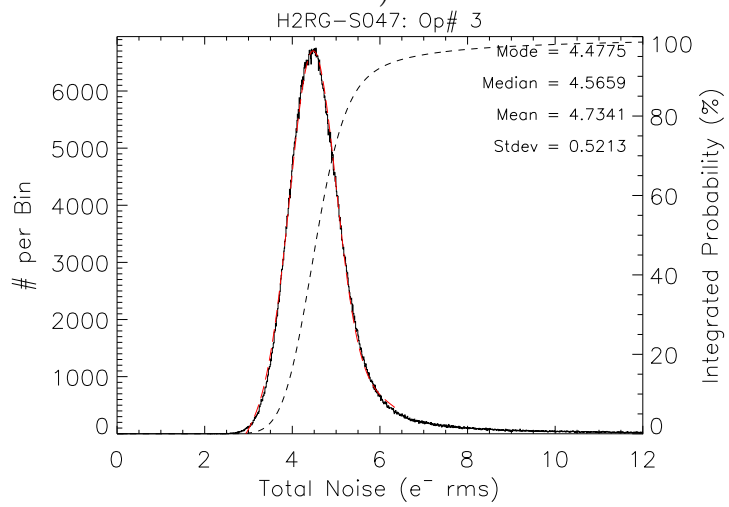

d)

Figure 5. Total noise per output off flight candidate SCA H2RG-S047. These per-pixel histograms were made using a set of 50 EXPTIME $=892 s$ MULTI $22 \times 4$ sampled integrations.

\section{SUMMARY}

Table 3 summarizes the dark performance data of all $\lambda_{\text {co }}=5 \mu \mathrm{m}$ HAWAII-2RG parts discussed in this article. The dark performance of all 3 parts is excellent, meeting NIRSpec's $<6 e^{-}$rms per EXPTIME $=1008 s$ MULTI-22×4 (88 up-the-ramp samples) integration total noise requirement with $10 \%-20 \%$ margin. NIRSpec SCA production and testing are ongoing at TIS, and this article represents a snapshot of the best available SCAs as of June, 2008. Although we are pleased with the dark performance of these SCAs so far, it is possible that the actual flight parts may out perform what is reported here.

The conversion gains used in this paper were computed using the noise modeling technique (Section 3.3.2). While these conversion gains are consistent with the noise properties of the data, and also previous testing of similar parts under similar operating conditions by other groups, we plan to continue validating this technique. For this reason, there is a risk that some of the noise and dark current measurements reported here may change somewhat as more is learned about computing conversion gain at the relevant ultra-low background light levels. We anticipate that any such changes are likely to be no more than a few percent. 
Table 3. SCA Dark Performance Summary

\begin{tabular}{|c|c|c|c|c|c|}
\hline \multirow[b]{3}{*}{ Parameter } & \multirow[b]{3}{*}{ Unit } & \multirow[b]{3}{*}{ Requirement } & \multicolumn{3}{|c|}{ Measured Value } \\
\hline & & & EN & FC- & $\mathrm{FC}$ \\
\hline & & & S042 & S044 & $\mathrm{S} 047$ \\
\hline Dark current & $e^{-} s^{-1}$ pixel $^{-1}$ & $<0.01$ & 0.0097 & 0.0098 & 0.0076 \\
\hline Noise per $\mathrm{CDS}^{b}$ & $e^{-} \mathrm{rms}$ & $\mathrm{N} / \mathrm{A}$ & 17.3 & 16.9 & 14.8 \\
\hline Total noise & $e^{-} \mathrm{rms}$ & & & & \\
\hline EXPTIME $=892.08 \mathrm{~s}$ & & $\mathrm{~N} / \mathrm{A}$ & 5.35 & 5.27 & 4.62 \\
\hline EXPTIME $=1008 s$ & & $<6$ & $5.46^{c}$ & $5.39^{c}$ & $4.73^{c}$ \\
\hline Transimpedence gain & $\mu \mathrm{V} / \mathrm{e}^{-}$ & & & & \\
\hline Dark & & $\mathrm{N} / \mathrm{A}$ & 3.83 & 3.98 & 3.85 \\
\hline
\end{tabular}

${ }^{a}$ Includes any ROIC glow from 88 up-the-ramp samples.

${ }^{b}$ Includes read noise in the test setup.

${ }^{c}$ Computed by test and analysis. The test inputs are the measured dark current and total noise per EXPTIME $=892.08 s$ MULTI-22 $\times 4$ integration, as specified in this table. Noise was modeled using Equation 12. 


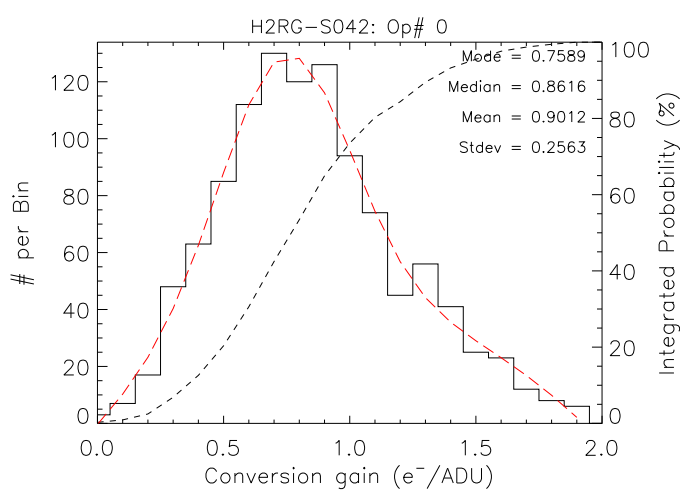

a)

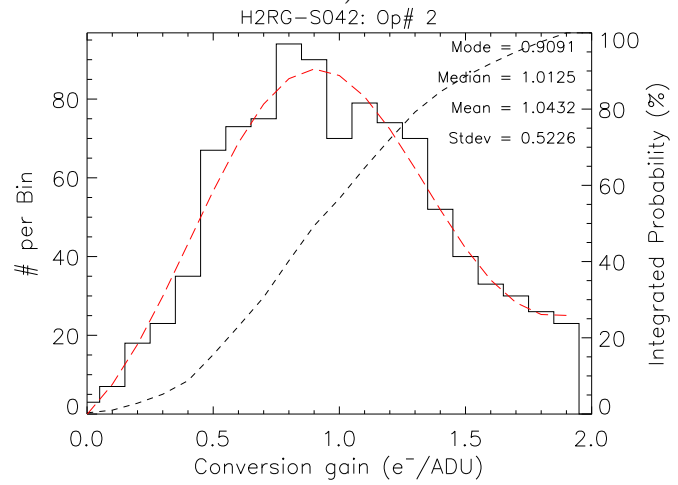

c)

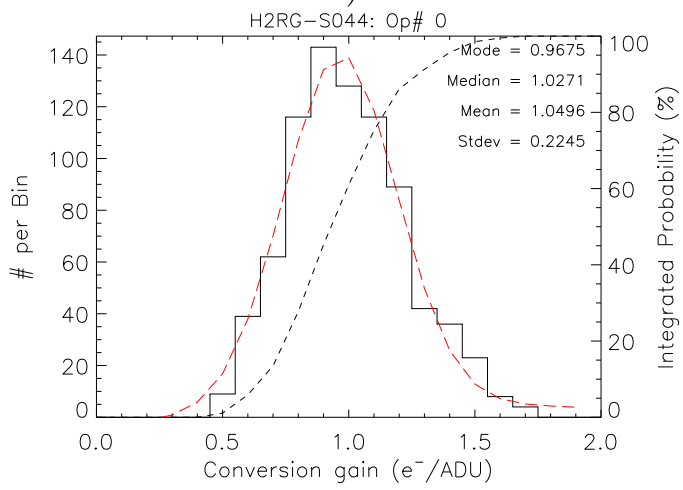

e)

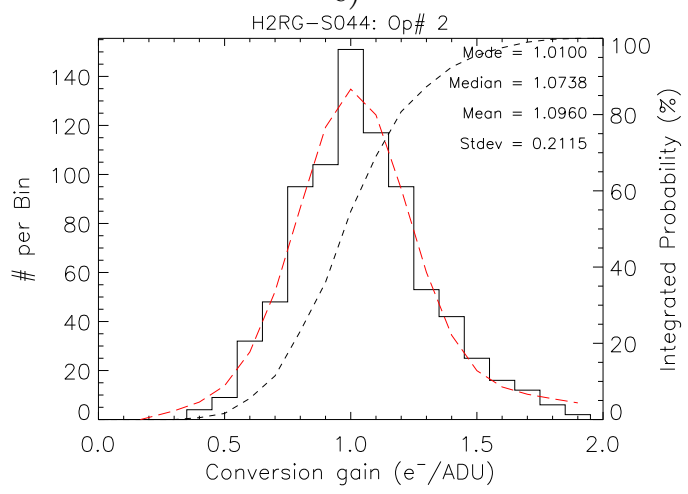

g)

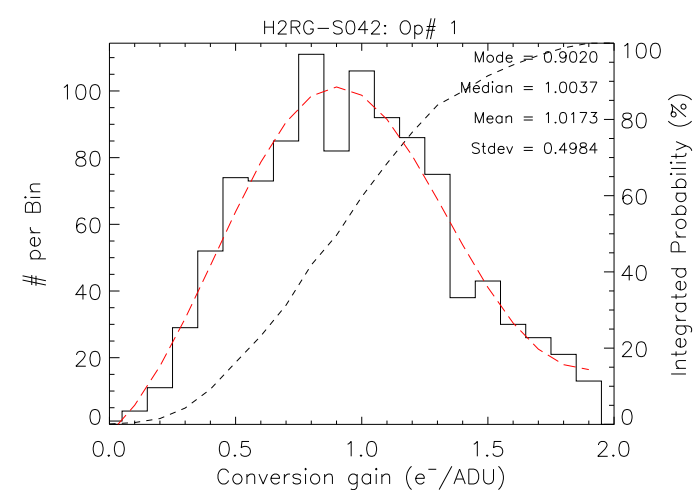

b)

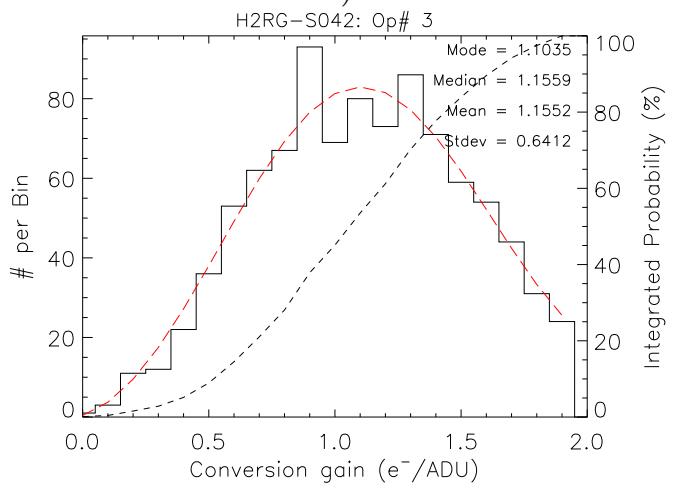

d)

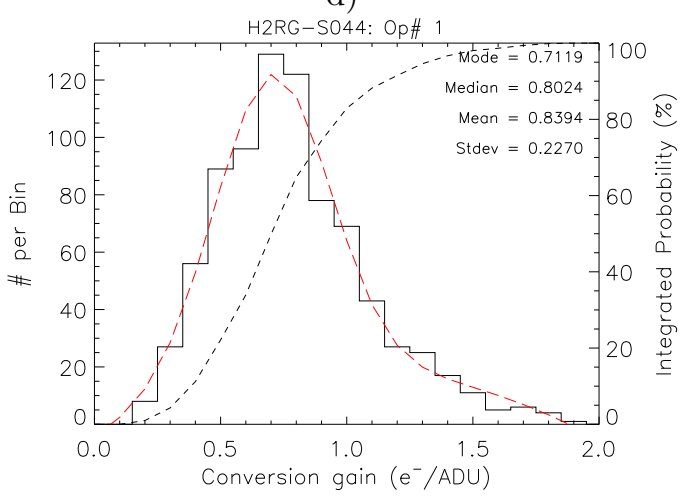

f)

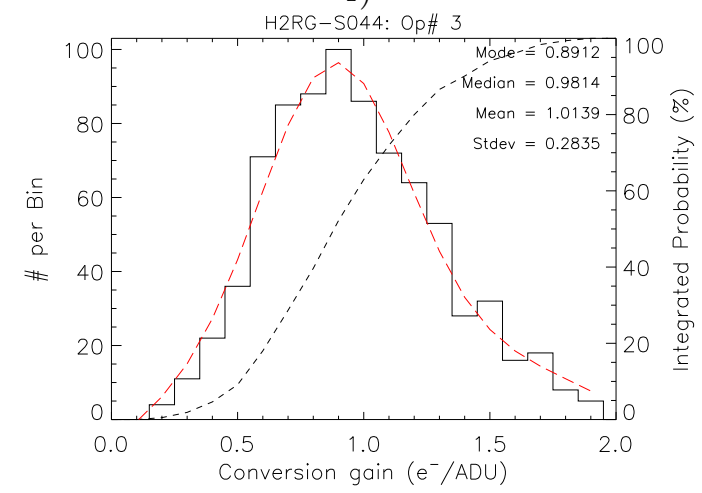

h)

Figure 6. Histograms of $g_{c}$ computed by noise modeling. 


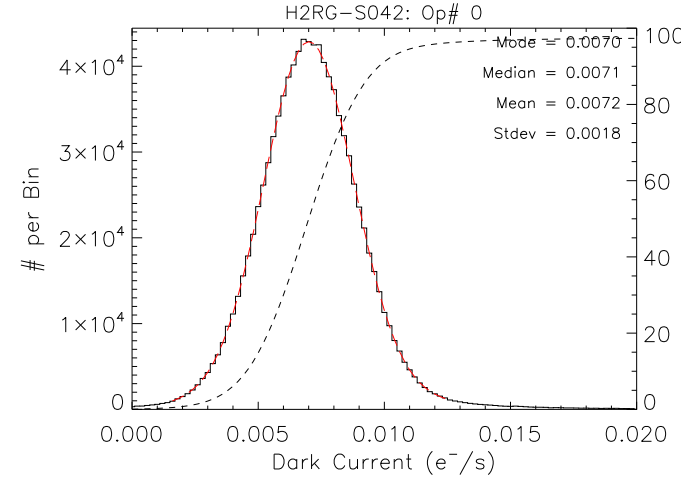

a)

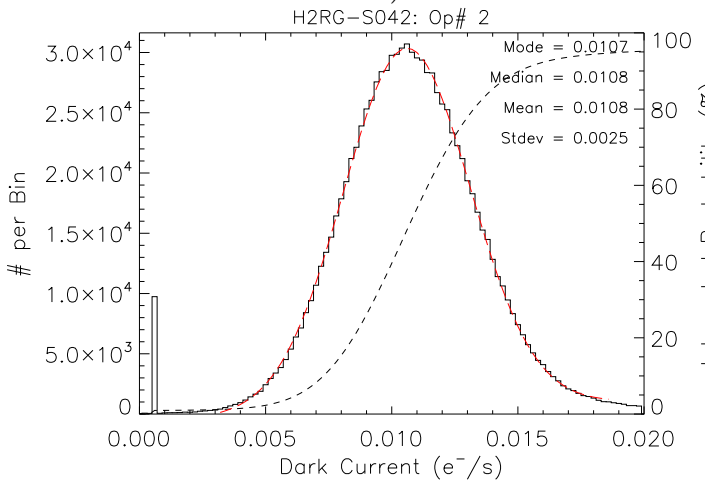

c)

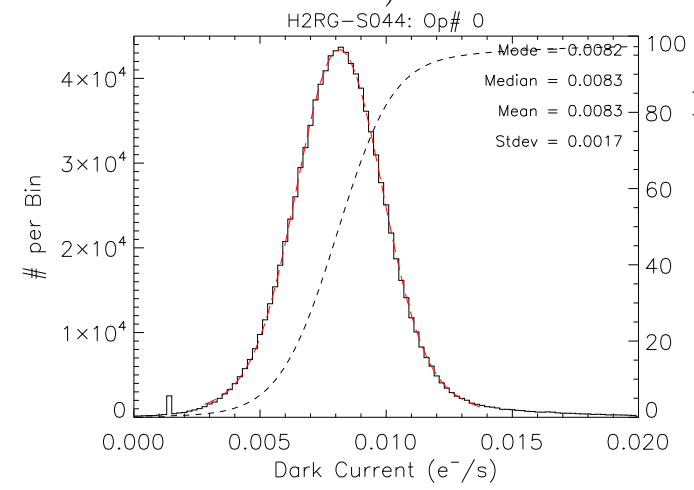

e)

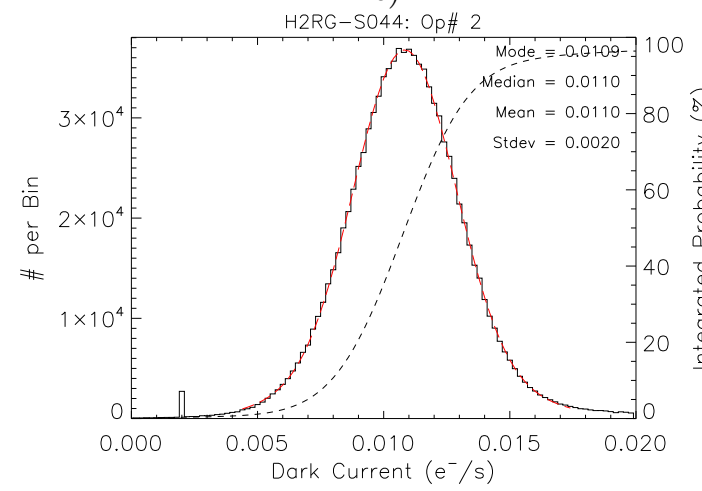

g)

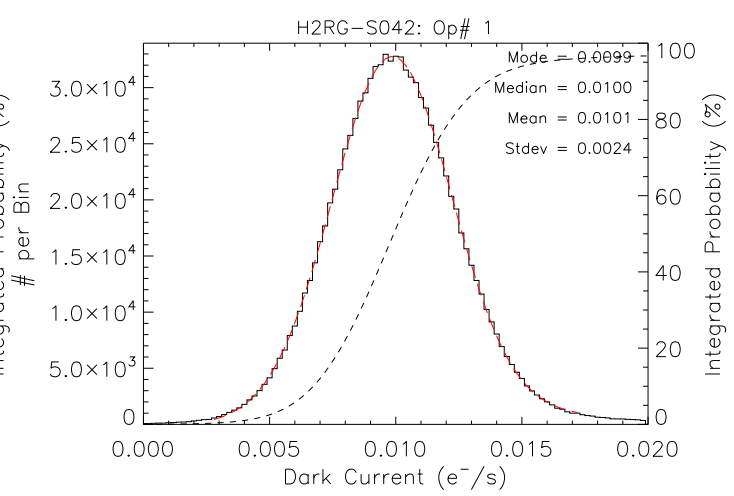

b)

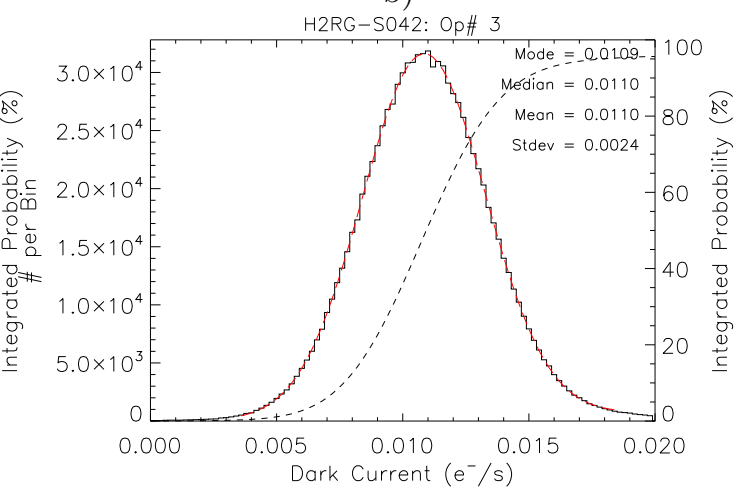

d)

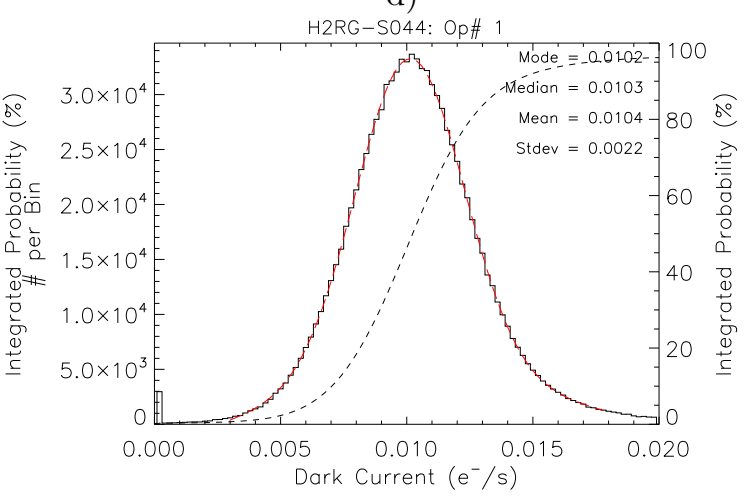

f)

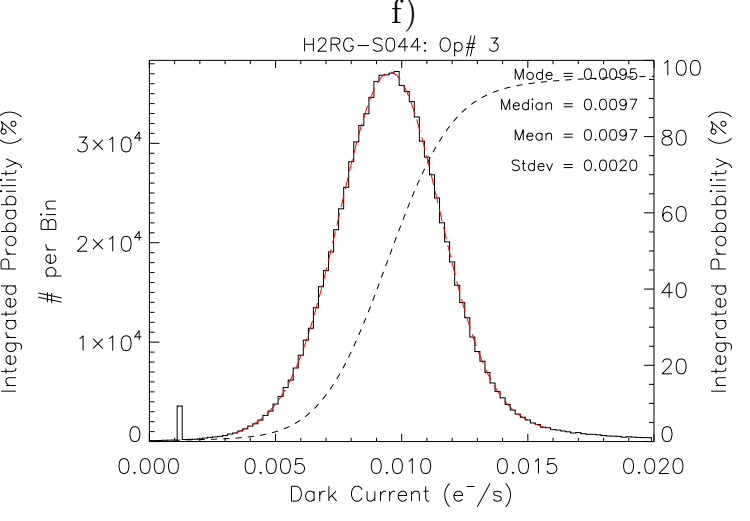

h)

Figure 7. Dark current per output. 


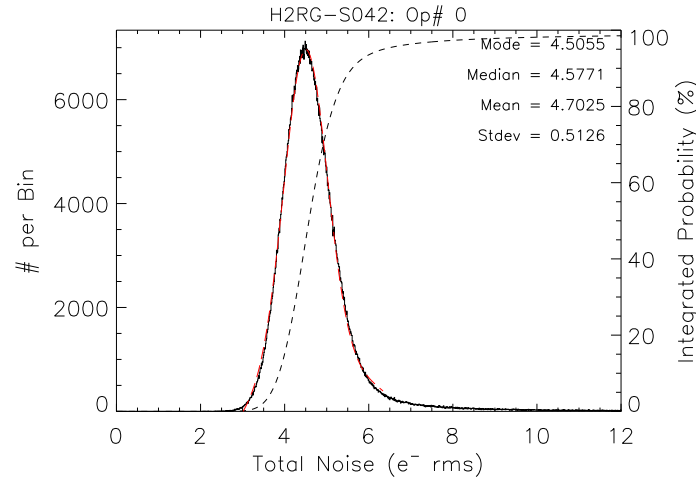

a)

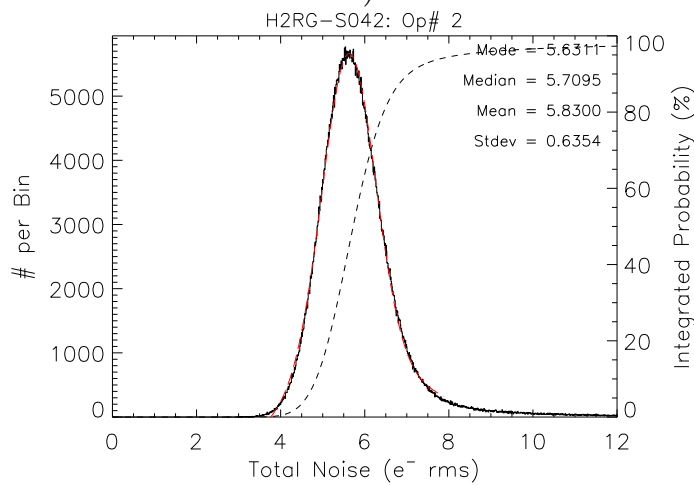

c)

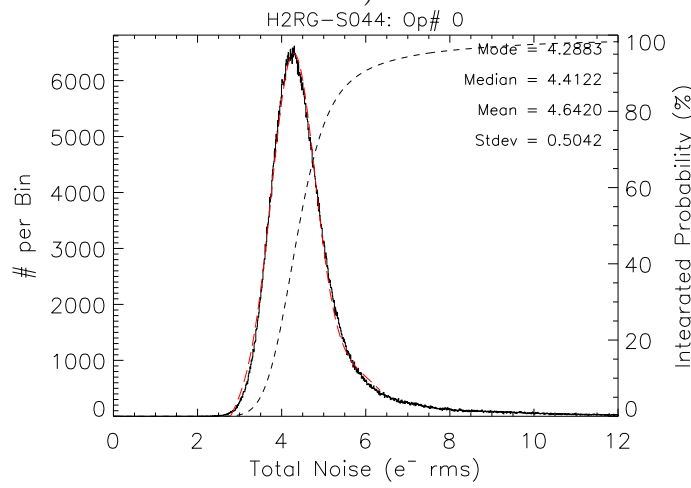

e)

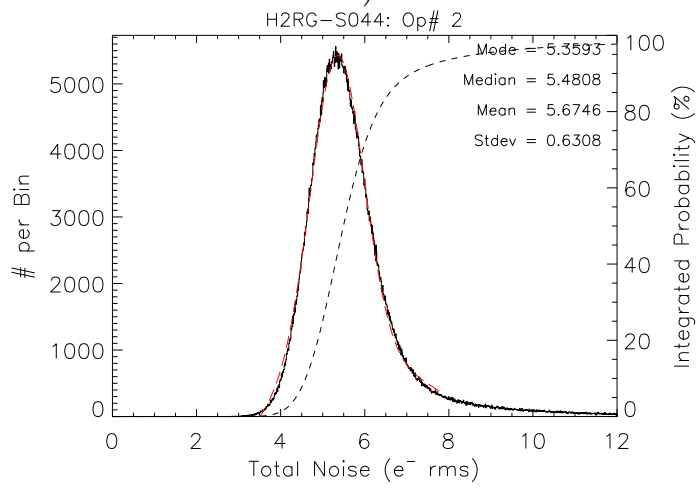

g)

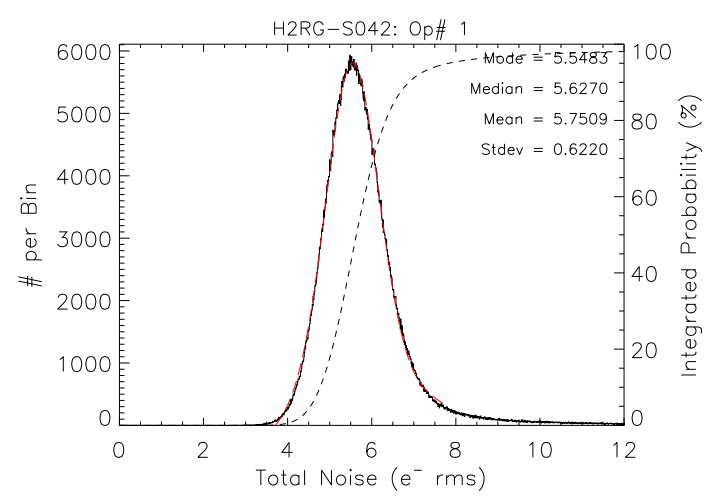

b)

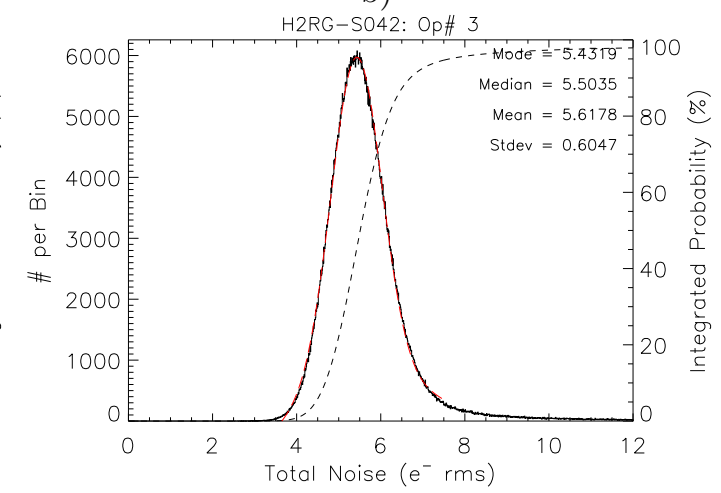

d)

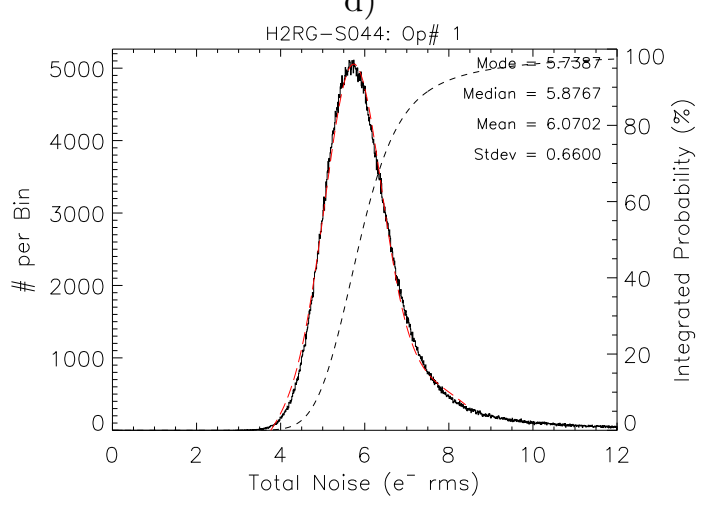

f)

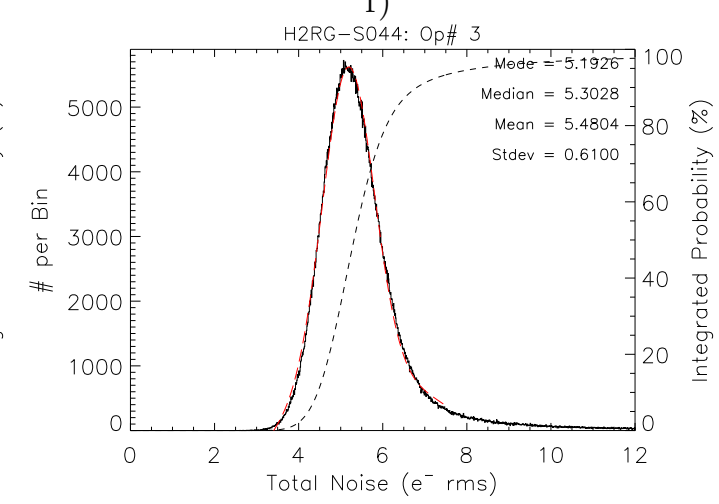

h)

Figure 8. Total noise per output, EXPTIME $=892.08 s$. 


\section{ACKNOWLEDGMENTS}

This work was supported by NASA and ESA as part of the James Webb Space Telescope Project. Ori Fox wishes to thank NASA's Graduate Student Researcher Program for a grant to the University of Virginia.

\section{REFERENCES}

[1] Rauscher, B. J., Figer, D. F., Regan, M. W., Boeker, T., Garnett, J., Hill, R. J., Bagnasco, G., Balleza, J., Barney, R., Bergeron, L. E., Brambora, C., Connelly, J., Derro, R., DiPirro, M. J., Doria-Warner, C., Ericsson, A., Glazer, S. D., Greene, C., Hall, D. N., Jacobson, S., Jakobsen, P., Johnson, E., Johnson, S. D., Krebs, C., Krebs, D. J., Lambros, S. D., Likins, B., Manthripragada, S., Martineau, R. J., Morse, E. C., Moseley, S. H., Mott, D. B., Muench, T., Park, H., Parker, S., Polidan, E. J., Rashford, R., Shakoorzadeh, K., Sharma, R., Strada, P., Waczynski, A., Wen, Y., Wong, S., Yagelowich, J., and Zuray, M., "Detectors for the James Webb Space Telescope near-infrared spectrograph," in [Optical, Infrared, and Millimeter Space Telescopes. Edited by Mather, John C. Proceedings of the SPIE, Volume 5487, pp. 710-726 (2004).], Mather, J. C., ed., Presented at the Society of Photo-Optical Instrumentation Engineers (SPIE) Conference 5487, 710-726 (Oct. 2004).

[2] Rauscher, B. J., Böker, T., Cabelli, C., De Marchi, G., Ferruit, P., Garnett, J., Hill, R. J., Loose, M., Regan, M. W., Waczynski, A., Wen, Y., Wong, S., Zandian, M., Alexander, D., Brambora, C. K., Derro, R., Dunn, C., Ellis, T., Garrison, M. B., Howe, B., Jakobsen, P., Johnson, T. E., Jurado, M., Lee, G., Manthripragada, S. S., Marsh, J. M., Marshall, C., Martineau, R. J., Mott, B., Nieznanski, J., Roher, W. D., Shakoorzadeh, K. B., Smith, M. T., Strada, P., Wallis, P., Xia-Serafino, W., and York, J., "Detectors for the James Webb Space Telescope near infrared spectrograph (NIRSpec)," in [Space Telescopes and Instrumentation I: Optical, Infrared, and Millimeter. Edited by Mather, John C.; MacEwen, Howard A.; de Graauw, Mattheus W. M.. Proceedings of the SPIE, Volume 6265, pp. 626538 (2006).], Presented at the Society of PhotoOptical Instrumentation Engineers (SPIE) Conference 6265 (July 2006).

[3] Rauscher, B. J., Fox, O., Ferruit, P., Hill, R. J., Waczynski, A., Wen, Y., Xia-Serafino, W., Mott, B., Alexander, D., Brambora, C. K., Derro, R., Engler, C., Garrison, M. B., Johnson, T., Manthripragada, S. S., Marsh, J. M., Marshall, C., Martineau, R. J., Shakoorzadeh, K. B., Wilson, D., Roher, W. D., Smith, M., Cabelli, C., Garnett, J., Loose, M., Wong-Anglin, S., Zandian, M., Cheng, E., Ellis, T., Howe, B., Jurado, M., Lee, G., Nieznanski, J., Wallis, P., York, J., Regan, M. W., Hall, D. N. B., Hodapp, K. W., Böker, T., De Marchi, G., Jakobsen, P., and Strada, P., "Detectors for the James Webb Space Telescope Near-Infrared Spectrograph. I. Readout Mode, Noise Model, and Calibration Considerations," PASP 119, 768-786 (July 2007).

[4] Rauscher, B. J., Alexander, D., Brambora, C. K., Derro, R., Engler, C., Fox, O., Garrison, M. B., Henegar, G., Hill, R. J., Johnson, T., Lindler, D. J., Manthripragada, S. S., Marshall, C., Mott, B., Parr, T. M., Roher, W. D., Shakoorzadeh, K. B., Smith, M., Waczynski, A., Wen, Y., Wilson, D., Xia-Serafino, W., Cabelli, C., Cheng, E., Garnett, J., Loose, M., Zandian, M., Zino, J., Ellis, T., Howe, B., Jurado, M., Lee, G., Nieznanski, J., Wallis, P., York, J., Regan, M. W., Bagnasco, G., Böker, T., De Marchi, G., Ferruit, P., Jakobsen, P., and Strada, P., "Detector arrays for the James Webb Space Telescope near-infrared spectrograph," in [Focal Plane Arrays for Space Telescopes III. Edited by Grycewicz, Thomas J.; Marshall, Cheryl J.; Warren, Penny G.. Proceedings of the SPIE, Volume 6690, pp. 66900M (2007).], Presented at the Society of Photo-Optical Instrumentation Engineers (SPIE) Conference 6690 (Sept. 2007).

[5] Mott, D. B. et al., "Characterization of the detector subsystem for near infrared spectrograph (nirspec) on the james webb space telescope," in [Proceedings of the SPIE, in press.], Presented at the Society of Photo-Optical Instrumentation Engineers (SPIE) Conference (2008).

[6] Fox, O., Waczynski, A., Wen, Y., Foltz, R. D., Hill, R. J., Kimble, R. A., Malumuth, E., and Rauscher, B. J., " ${ }^{55} \mathrm{Fe}$ X-ray Energy Response of Mercury Cadmium Telluride Near-Infrared Detector Arrays," in [Proceedings of the SPIE, in press.], Presented at the Society of Photo-Optical Instrumentation Engineers (SPIE) Conference (2008).

[7] Bacon, C. M., McMurtry, C. W., Pipher, J. L., Forrest, W. J., and Garnett, J. D., "Burst noise in the HAWAII-1RG multiplexer," in [Focal Plane Arrays for Space Telescopes II. Edited by Grycewicz, Thomas J.; Marshall, Cheryl J. Proceedings of the SPIE, Volume 5902, pp. 116-127 (2005).], Grycewicz, T. J. 
and Marshall, C. J., eds., Presented at the Society of Photo-Optical Instrumentation Engineers (SPIE) Conference 5902, 116-127 (Aug. 2005).

[8] Moore, A. C., Ninkov, Z., and Forrest, W. J., "Interpixel capacitance in nondestructive focal plane arrays," in [Focal Plane Arrays for Space Telescopes. Edited by Grycewicz, Thomas J.; McCreight, Craig R. Proceedings of the SPIE, Volume 5167, pp. 204-215 (2004).], Grycewicz, T. J. and McCreight, C. R., eds., Presented at the Society of Photo-Optical Instrumentation Engineers (SPIE) Conference 5167, 204-215 (Jan. 2004).

[9] Moore, A. C., Ninkov, Z., and Forrest, W. J., "Quantum efficiency overestimation and deterministic cross talk resulting from interpixel capacitance," Optical Engineering 45(7), 076402 (2006).

[10] Janesick, J., Klaasen, K., and Elliott, T., "CCD charge collection efficiency and the photon transfer technique," in [Solid state imaging arrays; Proceedings of the Meeting, San Diego, CA, August 22, 23, 1985 (A87-10977 01-35). Bellingham, WA, Society of Photo-Optical Instrumentation Engineers, 1985, p. 7-19. NASA-supported research.], Dereniak, E. L. and Prettyjohns, K. N., eds., Presented at the Society of PhotoOptical Instrumentation Engineers (SPIE) Conference 570, 7-19 (Jan. 1985). 